\title{
NOUVELLE
}

\section{Le récepteur proapoptotique OXIR dans le cancer colique}

\section{Silencieux in situ mais activable en thérapie}

Marc Laburthe
Centre de recherche biomédicale Bichat-Beaujon (CRB3), UMRS 773 Inserm, université Paris-Diderot,

faculté de médecine Xavier Bichat, 16, rue Henri Huchard, 75018 Paris, France.

marc.laburthe@inserm.fr
$>$ Le cancer colorectal est l'un des plus fréquents en Europe et en Amérique du Nord. L'exérèse de la tumeur primaire, voire de certaines métastases, est associée à une chimiothérapie dont la molécule de référence est le 5-fluorouracile ( $5 \mathrm{FU})$ souvent utilisé en combinaison avec d'autres agents. La chimiothérapie reste cependant peu efficace dans les stades avancés. La génétique moléculaire a permis de décrire des gènes clés (oncogènes, gènes suppresseurs de tumeurs) dont la mutation ou l'expression altérée peut conduire au cancer colorectal. Par ailleurs, on sait que la prolifération des cellules cancéreuses coliques est stimulée par des facteurs de croissance présents dans leur environnement parmi lesquels l'epidermal growth factor (EGF) joue un rôle important à côté de peptides hormonaux tels que la gastrine ou la neurotensine.

\section{L'inhibition de la croissance}

des cellules cancéreuses par les orexines La biologie des régulations cellulaires nous enseigne que tout mécanisme de contrôle requiert un équilibre entre des signaux stimulateurs et inhibiteurs, le yin et le Yang de la vie cellulaire. L'idée préconçue suivant laquelle les signaux d'inhibition de la croissance cellulaire (inhibition de la prolifération et/ou induction de la mort cellulaire) auraient disparu dans la cellule cancéreuse va de pair avec une absence de recherche de tels signaux. Partant de ce constat, nous avions recherché en 2004 de possibles inhibiteurs de la croissance de cellules cancéreuses coliques humaines parmi les peptides neurohormonaux existants [1]. Cette recherche a conduit à une découverte étonnante puisque deux peptides présents dans le cerveau, les orexines $A$ et $B$, sont de puissants inhibiteurs de la croissance de la lignée cancéreuse colique HT-29. Cette découverte est étonnante parce que ces peptides, produits par des neurones hypothalamiques, contrôlent de nombreuses fonctions centrales et en particulier le sommeil (Encadré 1), et n'ont a priori rien à voir avec les cancers coliques.

Dans un article récent [2], nous enrichissons ces observations initiales de

\section{Les orexines}

Les orexines $A$ et $B[4]$, aussi nommées hypocrétines 1 et 2 [5], sont codées par le même gène dans un précurseur commun. Ce sont deux peptides présents dans des neurones hypothalamiques qui se projettent dans de nombreuses aires cérébrales et contrôlent des fonctions variées dont le sommeil, la vigilance, la prise alimentaire, la respiration, le système de récompense ou l'addiction aux drogues [6]. Leur impact majeur sur le sommeil et la vigilance est révélé en pathologie puisqu'un déficit en orexine induit la narcolepsie (hypersomnolence et brusques épisodes de sommeil diurnes) associée à une cataplexie [6]. Les orexines ont des effets extracérébraux comme en témoignent l'existence de leurs récepteurs et/ou d'effets biologiques dans plusieurs organes périphériques [7]. La synthèse et la production d'orexines dans les tissus périphériques restent cependant non élucidées chez l'homme.

nouvelles données qui ouvrent des perspectives passionnantes concernant les orexines et le cancer colorectal. Nous montrons que les orexines inhibent la croissance de nombreuses lignées issues de cancers coliques primaires ou de métastases en provoquant la mort cellulaire par apoptose et cela en interagissant avec l'un des deux récepteurs des orexines, le récepteur OXIR. OXIR est un récepteur à sept domaines transmembranaires couplé à la protéine Gq (Encadré 2), qui est exprimé de façon aberrante dans les cancers colorectaux indépendamment de leur localisation et de leur stade. S'il est exprimé de façon aberrante c'est que les cellules épithéliales colorectales normales (qui donnent naissance

\section{Les récepteurs des orexines}

Les récepteurs $O X 1 R$ et $0 \times 2 R$ des orexines sont des récepteurs couplés aux protéines $\mathrm{G}(\mathrm{RCPG})$ appartenant à la famille de la rhodopsine. Couplés à la protéine $\mathrm{Gq}$, ils augmentent le calcium cytosolique [7]. La signalisation du récepteur $O X I R$ qui conduit à la réponse apoptotique vient d'être décrite et ne passe pas par cette voie calcique. En effet, elle implique la phosphorylation de deux tyrosines présentes dans des motifs de type immunoreceptor tyrosine-based inhibitory motif (ITIM) habituellement rencontrés dans les immunorécepteurs et très peu décrits dans les RCPG [8-10]. Cette phosphorylation aboutit à l'activation de la phosphatase Src homology phosphatase 2 (SHP2) qui déclenche la cascade apoptotique [8-10]. Ce mécanisme très original constitue un nouveau paradigme dans le signalisation des RCPG [10]. 
aux cellules cancéreuses) n'expriment pas du tout le récepteur OXIR. Cette expression aberrante est conservée dans les cellules cancéreuses ayant métastasé dans le foie, organe cible majeur des métastases de ce cancer. Cet article rapporte deux autres résultats majeurs: (1) les orexines sont capables d'induire la mort cellulaire par apoptose suivant la voie mitochondriale [1] dans des cellules cancéreuses coliques humaines sélectionnées pour être devenues insensibles à la chimiothérapie par le $5 \mathrm{FU}$; (2) dans un modèle de xénogreffe de cellules cancéreuses coliques humaines chez la souris nude, les orexines, administrées par voie intrapéritonéale à des doses qui n'entraînent aucun effet secondaire notable, empêchent le développement de tumeurs et, mieux encore, font régresser considérablement des tumeurs déjà établies [2].

\section{$O X 1 R$ : un gène suppresseur}

de tumeur silencieux chez l'homme

Puisque les orexines sont des neuropeptides naturels présents dans notre organisme, la question se pose de savoir pourquoi elles ne tuent pas les cellules cancéreuses coliques chez les malades. La réponse est sans doute que ces peptides ne sont pas présents dans le côlon humain normal ni dans les tumeurs colorectales, et cela même à des taux infimes puisque la technique de RT-PCR échoue à détecter des traces d'ARNm du précurseur des orexines [2]. Comme les orexines ne semblent pas être présentes à des taux significatifs dans le sang périphérique, il est légitime de conclure que le récepteur OXIR exprimé de façon aberrante dans les cancers coliques n'est tout simplement pas activé in vivo chez les malades. Paradoxalement, les malades ont dans leur cerveau une molécule naturelle très active contre la progression des tumeurs coliques mais qui ne leur sert à rien puisqu'elle n'accède jamais à la tumeur. Le récepteur OXIR des cellules cancéreuses coliques est donc un récepteur silencieux in situ. C'est un nouveau type de gène dans les cancers, un gène suppresseur de tumeur dont l'expression est induite (par un mécanisme encore inconnu) dans le cancer mais qui reste inactif faute d'activateur endogène sur le site de la tumeur. En revanche, si l'on apporte l'activateur de façon exogène, alors ce gène suppresseur de tumeur devient une cible thérapeutique très efficace. Cette situation est paradoxale puisque les cancers sont généralement associés à une perte de fonction de gènes suppresseurs de tumeur initialement présents dans la cellule normale qui donne naissance au cancer. Dans le cas présent, la cellule colique normale n'exprime pas ce gène suppresseur de tumeur mais il apparaît, sous une forme certes silencieuse, dans les cancers colorectaux et leurs métastases. Cette expression des récepteurs des orexines est-elle restreinte au cancer colorectal ? Cette question importante a déjà reçu un début de réponse puisque des lignées de neuroblastome [1] ou de cancer du pancréas [3] expriment respectivement des récepteurs $0 \times 1 R$ et $0 \times 2 R$ et sont sensibles à l'apoptose induite par les orexines. L'extension de ces données à d'autres cancers est en cours.

Un nouveau concept émerge donc, celui du néogène suppresseur de tumeur, silencieux mais activable en thérapie. Ces données ouvrent des perspectives dans le traitement pharmacologique des cancers colorectaux [2]. En cancérologie, comme dans d'autres domaines, il y a souvent loin de la coupe aux lèvres, et de nombreux travaux sont encore nécessaires pour passer, éventuellement, de ce nouveau concept à un traitement des cancers colorectaux chez l'homme. $\diamond$ How to turn a silent proapoptotic gene in a potent antitumoral target in colorectal cancer

\section{CONFLIT D'INTÉRÊTS}

L'auteur déclare n'avoir aucun conflit d'intérêts concernant les données publiées dans cet article.

\section{RÉFÉRENCES}

1. Rouet-Benzineb P, Rouyer-Fessard C, Jarry A, et al. Orexins acting at native $\mathrm{OX}(1)$ receptor in colon cancer cells and neuroblastoma cells or at recombinant $\mathrm{OX}(1)$ receptor suppress cell growth by inducing apoptosis. J Biol Chem 2004 ; 279 : 45875-86.

2. Voisin $T$, દl Firar A, Fasseu M, et al. Aberrant expression of $0 X 1$ receptors for orexins in colon cancers and liver metastases: an openable gate to apoptosis. Cancer Res $2011 ; 71: 3341-51$.

3. Voisin T, દl Firar A, Avondo V, Laburthe M. Orexininduced apoptosis: the key role of the seventransmembrane domain orexin type 2 receptor. Endocrinology $2006 ; 147$ : 4977-84.

4. De Lecea L, Kilduff TS, Peyron C, et al. The hypocretins: hypothalamus-specific peptides with neuroexcitatory activity. Proc Natl Acad Sci USA 1998 ; 95 : 322-7.

5. Sakurai T, Amemiya A, Ishi M, et al. Orexins and orexin receptors: a family of hypothalamic neuropeptides and $G$ protein-coupled receptors that regulate feeding behavior. Cell 1998; 92 : 573-85.

6. Mieda M, Sakurai T. Integrative physiology of orexins and orexin receptors. CNS Neurol Disord Drug Targets 2009 ; 4 : 281-95.

7. Voisin T, Rouet-Benzineb P, Reuter N, Laburthe M. Orexins and their receptors: structural aspects and role in peripheral tissues. Cell Mol Life Sci 2003 ; 60 : 72-87.

8. Voisin T, દl Firar A, Rouyer-Fessard C, et al. A hallmark of immunoreceptor, the tyrosine-based inhibitory motif ITIM, is present in the $G$ protein-coupled receptor OXIR for orexins and drives apoptosis: a novel mechanism. FASEB J 2008 ; 22 : 1993-2002.

9. El Firar A, Voisin T, Rouyer-Fessard C, et al. Discovery of a functional immunoreceptor tyrosine-based switch motif in a 7-transmembrane spanning receptor: role in the orexin receptor $0 X 1 \mathrm{R}$-driven apoptosis. FASEB J $2009 ; 23: 4069-80$.

10. Laburthe M, Voisin T. The orexin receptor OXIR in colon cancer: a promising therapeutic target and a new paradigm in $\mathrm{G}$ protein-coupled receptor signalling through ITIMs. Br J Pharmacol $2012 ; 165$ 1678-87.

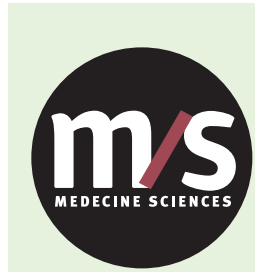

Tarifs d'abonnement M/S - 2012

Abonnez-vous

à médecine/sciences
> Grâce à $m / s$, vivez en direct les progrès des sciences biologiques et médicales

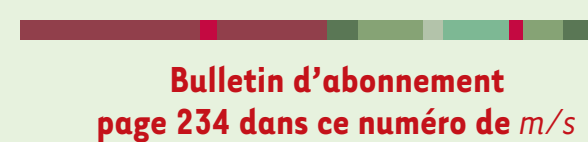

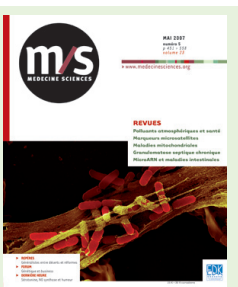

